\title{
Exotics at BESIII
}

\author{
Riccardo Farinelli ${ }^{1, *}$ \\ on behalf of the BESIII Collaboration \\ ${ }^{1}$ INFN Sez. Ferrara and University of Ferrara - Via Saragat 1, Ferrara
}

\begin{abstract}
With a collected integrated luminosity of about $12 \mathrm{fb}^{-1}$, useful data set for XYZ new resonances, BESIII continues the exploration of these exotic charmonium-like states. In this proceeding, recent results on the measurements of the line-shape of $e^{+} e^{-} \rightarrow \pi^{+} \pi^{-} \psi(3868)$ and $\pi^{+} D^{0} D^{*-}$, as well as the $\mathrm{J}^{P}$ determination of $Z_{C}(3900)$ will be presented. An abundance of $Y$ will be reported in several decay channels. Finally the present BESIII data above the $e^{+} e^{-} \rightarrow \Lambda_{C} \bar{\Lambda}_{C}$ threshold will be shortly discussed because they are barely in agreement with the current Belle data on the candidate charmed baryonium state $Y(4660)$. A BEPCII upgrade, that will allow to settle this open question, has been funded.
\end{abstract}

\section{Introduction}

Strong interaction is one of the four fundamental forces and it acts on elementary particles such as quarks. Quarks are bound together into hadrons following strict rules given by color quantum number. Only certain combination of bound states are allowed: they are classified baryons, bound states with three quarks or mesons with a quark and an anti-quark. The strong interaction does not forbid others kinds of bound states but up to a few years ago only those two categories had been discovered. In the past years physicists searched for other kinds of bound states such as glueball, tetraquark or pentaquark. In the last years two discoveries of interest have to be cited: the $Z(3900)$ [1] discovered by BESIII is a tetraquark candidate and the $P_{c}^{+}[2]$ discovered by $\mathrm{LHCb}$ is referred as pentaquark.

In the heavy meson sector, the potential model describes the bound states of charm and anti-charm quarks, called charmonium, and it can predict the mass and width of the bound state, e.g. $\eta_{c}, \psi$ and $\chi_{c}$ particles as a function of its quantum number. Charmonium state masses range from about $4.0 \mathrm{GeV}$ to $4.4 \mathrm{GeV}$ and the energy region of interest is divided by the production threshold of the $D$ meson around $3.74 \mathrm{GeV}$ : below this energy the charmonium decays only to in un-charmed baryons, other charmonium states. Once the mass of the charmonium states is above $3.74 \mathrm{GeV}$ then they decay mostly in $D \bar{D}$ [3]. The theoretical prediction of the potential model and the measured masses of the charmonium states are in agreement below the open charm threshold. Above this energy it was expected to discover the excited states of the charmonium states but only a few have been measured and many others have not been found.

Since 2003 many states have been discovered around this energy region, some of those show connections to the charmonium but they can not be described by charmonium states.

\footnotetext{
*e-mail: rfarinelli@fe.infn.it
} 
First of all the $X(3872)$ [4] observed by Belle in the decay channel $J / \psi \pi^{+} \pi^{-}$is manifestly exotic due to its width, smaller than $1.2 \mathrm{MeV} / \mathrm{c}^{2}$, and because it violates its quantum number in certain decay [5]. Later on in $2005 \mathrm{BaBar}$ observed the $Y$ (4260) [6], a structure with quantum number $1^{--}$that does not match with any $J / \psi$ excitation and populates an energy region with an abundance of states with the same quantum number. While BESIII was studying the $Y(4260)$ decay, another particle with a clear exotic behaviour shows up: the Z(3900) [1], a resonance decaying in $J / \psi$ a charmonium state and a charged pion. The $Z(3900)$ is the first evidence of a new kind of bound state that it is not neither a meson nor a baryon. Those $X Y Z$ states have been labelled with different letters to define different behaviors: $Y$ states have quantum number $1^{--}$while $Z$ states are connected to this possible tetraquark explanation. All of them decay in charmonium states and some of them show connection such as the $Y(4260)$ which is connected to both the $X(3872)$ with a radiative transition [7] and the Z(3900) with an hadronic transition [1]. Figure 1 shows the charmonium spectrum as a function of quantum number and the discovered exotic states.

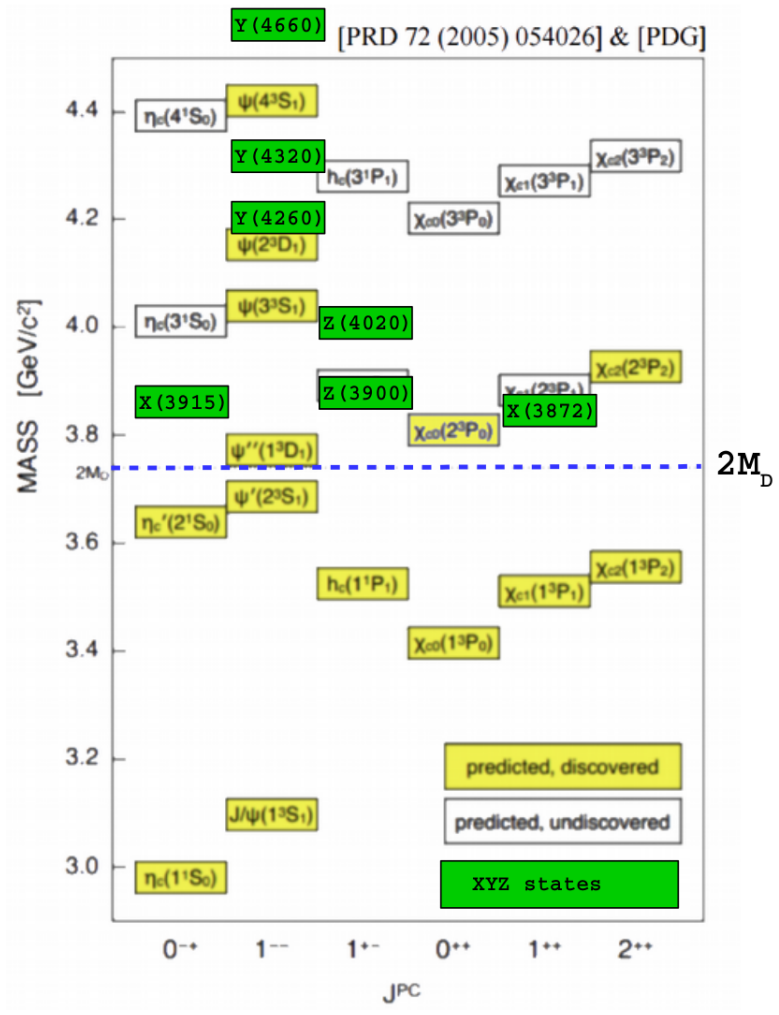

Figure 1. The charmonium spectrum in the mass (vertical axis) vs quantum number (horizontal axis) plane. $X Y Z$ are also represented in order to show graphically the abundance in the considered energy region.

\section{The BESIII experiment}

The BEijing Spectrometer (BESIII) is a experiment of high energy physics that measures the interaction results of the Beijing Electron Positron Collider (BEPCII) working in the center 
of mass energy between 2 and $4.6 \mathrm{GeV}$ [8]. The detector is built around the interaction point with a cylindrical symmetry given by a barrel and two end-caps. It is composed by a multilayer drift chamber that surrounds the beam pipe then there is a time-of-flight system and an electromagnetic calorimeter. Those detectors are placed inside a $1 \mathrm{~T}$ superconducting magnet. The muon identifier is placed in the return yoke of the solenoidal magnet. The geometrical coverage is $93 \%$ of the solid angle.

The energy range achieved by BEPCII constrains most of BESIII studies in the charmed region and BESIII accumulated during the years the world largest sample of $J / \psi$ and $\psi(3686)$. Thanks to the electron and positron beam, BESIII produces directly particles of quantum number $\mathrm{J}^{P C}=1^{--}$then it is a suitable experiment to study the $Y$ states because it can produce them directly.

\section{The $Z$ states}

After the discovery of the first $Z$ particle that has been claimed as tetraquark. the BESIII collaboration opened a new field of research to understand the nature of this particle. It has been observed in the decay channel $e^{+} e^{-} \rightarrow\left(J / \psi \pi^{+}\right) \pi^{-}$and since it decays in charmonium plus a charged pion it should contain at least four quarks: a $c \bar{c}$ and a $u \bar{d}$. Its mass is $3899 \pm$ $3.6 \pm 4.9 \mathrm{MeV} / \mathrm{c}^{2}$ and its width is $46 \pm 10 \pm 20 \mathrm{MeV} / \mathrm{c}^{2}$, the first error is statistical and the second is systematic [1]. Several theoretical papers described this resonance as a tetraquark, a molecular state or a charmonium hybrid $[9,10]$. The resonance has been confirmed by Cleo-C and Belle.

In 2015 the same decay with neutral pions $e^{+} e^{-} \rightarrow\left(J / \psi \pi^{0}\right) \pi^{0}$ shows a resonance with a mass and a width close to the charged mode ones [11]. This established the isospin triplet for this resonance. In order to understand the nature of this particle the measurement of its quantum number is needed and in 2017 the likelihood method has been used to support the quantum number $1^{+}$with a significance of $7 \sigma$ with respect the others [12].

Between 2013 and 2018 many other $Z$ states have been discovered. Some of them have been observed in the invariant mass spectrum of a pion plus a charmonium state such as the previous channel, or with an $h_{C}$ or $\psi(3686)$ [13-15]; others decays into $D$ meson or its excitation. Examples are $e^{+} e^{-} \rightarrow \pi^{+}\left(D \bar{D}^{*}\right)^{-}$or $e^{+} e^{-} \rightarrow \pi^{+}\left(D^{*} \bar{D}^{*}\right)^{-}$[16-18]. Those measurements indicate that the $Z$ state decay both in charmonium and in $D$ meson states and the masses of those resonances show two values of accumulation: $3900 \mathrm{MeV} / \mathrm{c}^{2}$ in $\pi J / \psi$ and in $D \bar{D}^{*}$ modes, while $\pi h_{C}$ and $D^{*} \bar{D}^{*}$ modes report a mass of about $4020 \mathrm{MeV} / \mathrm{c}^{2}$. The quantum number of $Z(3885) \rightarrow D D^{*}$ has been measured to be $1^{+}$and it is in agreement with the one measured in $Z(3900) \rightarrow \pi J / \psi$. Summary plots are shown in Fig. 2.

\section{The $Y$ states}

The research of the $Y$ states has began with BaBar [6] through the ISR technique. The $Y(4260)$ decays into $\pi^{+} \pi^{-} J / \psi$ and it has been confirmed by Belle and Cleo-C. BESIII can produce directly this resonance and thank to variation of few $\mathrm{MeV}$ it can scan the cross section of this process with an higher resolution with respect the ISR technique. In 2017 BESIII measured the cross section of this decay [19] using two different data-sets and two resonances describe the data with a significance greater than $7.6 \sigma$. This is a different result from the one of BaBar or Belle that had fit the cross section lineshape with a single resonance that was not able to describe perfectly the data. The resonances claimed by BESIII have masses of about 4222 and $4320 \mathrm{MeV} / \mathrm{c}^{2}$ and width of about 44.1 and $101.4 \mathrm{MeV} / \mathrm{c}^{2}$. The $Y(4320)$ has been observed for the first time in this decay channel and its mass is compatible with the $Y$ 


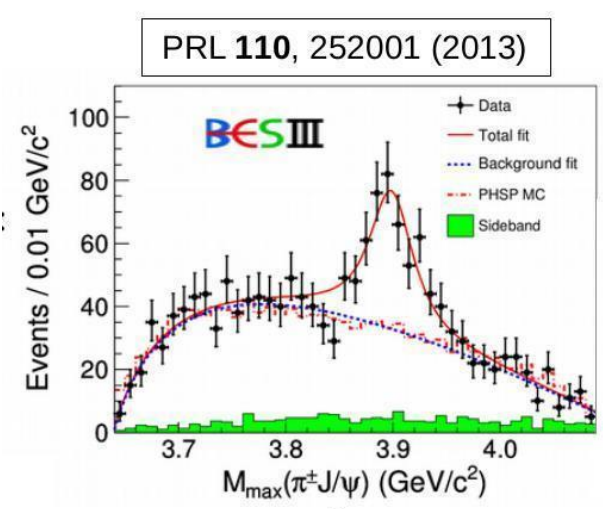

(a)

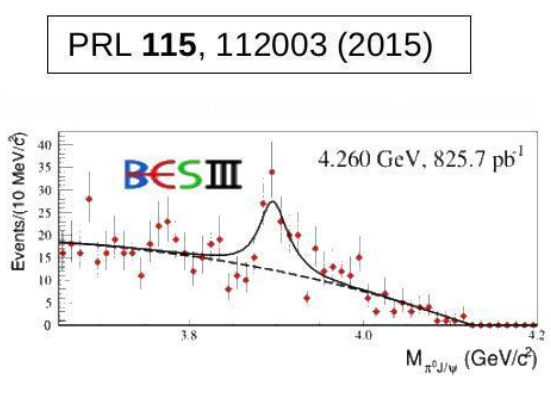

(b)

PRL 119, 072001 (2017)

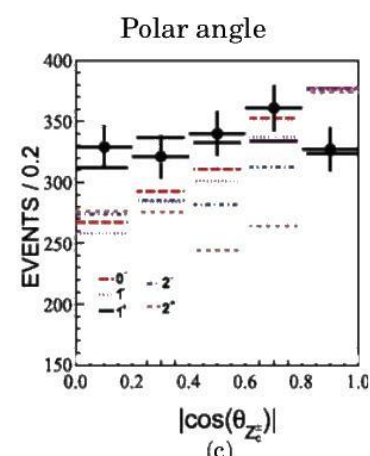

(c)

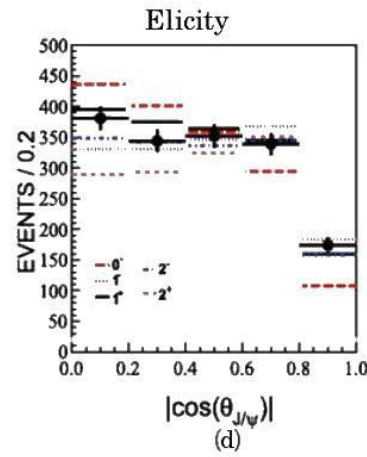

(d)

Figure 2. Summary of the plots of interest for the $Z(3900)$. On the top the invariant mass spectrum of the $Z(3900)$ with a charged (neutral) pion is shown ( $\mathrm{a}$ and $\mathrm{b}$ ). On the bottom the polar angle and elicity distribution used to define its quantum number (c and d).

measured by BaBar and Belle in $\pi \pi \psi(3686)$ decay channel.

A similar behavior has been observed in 2017 in the decay channel $e^{+} e^{-} \rightarrow \pi^{+} \pi^{-} h_{C}$ [20]. The $h_{C}$ has been reconstructed in $h_{C} \rightarrow \gamma \eta_{C}$ and $\eta_{C}$ has been reconstructed in 16 different modes. The cross section of the process can be described with two resonances too. This study reports masses of 4218 and $4391 \mathrm{MeV} / \mathrm{c}^{2}$. Within the errors this $Y(4218)$ is compatible with the previous $Y(4222)$. It is important to report also the observation of two resonances in the decay channel $e^{+} e^{-} \rightarrow \pi^{+} D D^{*-}$ that shows the presence of two resonances at the mass of 4228 and $4404 \mathrm{MeV} / \mathrm{c}^{2}$. The values are not totally compatible but they indicate and abundance of $Y$ states in this energy region.Summary plots are shown in Fig. 3.

Looking at the cross section of $e^{+} e^{-} \rightarrow \pi^{+} \pi^{-} \psi(3686)$ BaBar and Belle saw two resonances in the mass spectrum: $Y(4360)$ and $Y(4660)$ [21, 22]. BESIII could confirm the line-shape of the $Y(4360)$ and it measured two resonances with masses 4209 and $4383 \mathrm{MeV} / \mathrm{c}^{2}$ [23]. Due to its limit in the beam energy BESIII could not confirm the $Y(4660)$.

Before entering next section it is important to summarize those $Y$ states: BESIII observed two 
resonances with similar masses and widths in the energy region between 4.1 and $4.5 \mathrm{GeV}$ in three modes with charmonium and one with open charm mesons, as shown is Fig. 3.
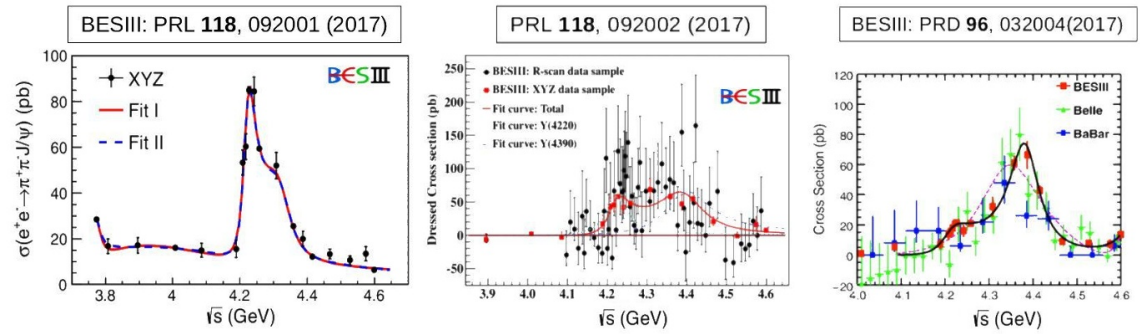

Figure 3. Cross section of the the Y process of interest: $\pi^{+} \pi^{-} J / \psi$ on the left, $\pi^{+} \pi^{-} h_{C}$ in the middle and $\pi^{+} \pi^{-} \psi(3686)$ on the right.

\section{The key particle: $\mathrm{Y}(4660)$}

The $Y(4660)$ is a resonance observed by Belle and BaBar in $e^{+} e^{-} \rightarrow \pi^{+} \pi^{-} \psi(3686)$ by means of ISR [21, 22]. BaBar reports a mass of about $4669 \mathrm{MeV} / \mathrm{c}^{2}$ and Belle $4652 \mathrm{MeV} / \mathrm{c}^{2}$. The cross section in the resonance peak is around $30 \mathrm{pb}$. Moreover BaBar performed a similar study in the decay channel $e^{+} e^{-} \rightarrow \pi^{+} \pi^{-} J / \psi$ [24] but in this mode there is no evidence of the $Y(4660)$ state despite this would be expected to be larger if $Y(4660)$ is a $c \bar{c}$ state. According to BaBar data [25] as elaborated in [26], at $90 \%$ C.L. :

$$
\frac{B R\left[Y(4660) \rightarrow \pi^{+} \pi^{-} J / \psi\right]}{B R\left[Y(4660) \rightarrow \pi^{+} \pi^{-} \psi(3686)\right]}<0.46
$$

The $Y(4660)$ has neither been observed in open charm channels such as $D D, D D^{*}$ or $D^{*} D^{*}$ [3].

Belle collaboration observed a baryonic decay of the $Y(4660)$ into $\Lambda_{C} \bar{\Lambda}_{C}$ [27] with a mass of $4652 \pm 3.4 \mathrm{MeV} / \mathrm{c}^{2}$ and a width of $62.6 \pm 5.6 \mathrm{MeV} / \mathrm{c}^{2}$. The cross section at the peak is around $0.55 \mathrm{nb}$ and the lineshape follows a nontrivial trend. BESIII collaboration studied the cross section of $e^{+} e^{-} \rightarrow \Lambda_{C} \bar{\Lambda}_{C}$ up to $4.6 \mathrm{GeV}$ and it has no sufficient energy to confirm the entire line-shape [28], see Fig. 4 on the right. The trend reported from the two experiments seems different around the baryon production threshold. The cross section of $Y(4660)$ to baryons at peak is about $0.55 \mathrm{nb}$ and can be compared with the cross section of $e^{+} e^{-} \rightarrow p \bar{p}$ of about $0.8 \mathrm{nb}$ at threshold while the cross section to $\pi \pi J / \psi$ is about $0.04 \mathrm{nb}$. From those observations it is possible to conclude that the baryonic coupling of the $Y(4660)$ is 10 times greater than the mesonic coupling. Since there is not another mesonic decay with larger branching ratio than $\pi \pi J / \psi$ it is possible to describe the behavior of this state with the old G.C. Rossi, G. Veneziano, G.F. Chew paradigm [29, 30] of an hidden charm baryonium decay where mostly poping up from the vacuum a light quark pair and falling apart as a charmed baryon pair.

\section{Upgrades in BEPCII and BESIII}

The comprehension of the $Y(4660)$ is a key part of the $X Y Z$ scenario. This is reason why BESIII and BEPCII will be upgraded on both detector and accelerator sides. A new inner 

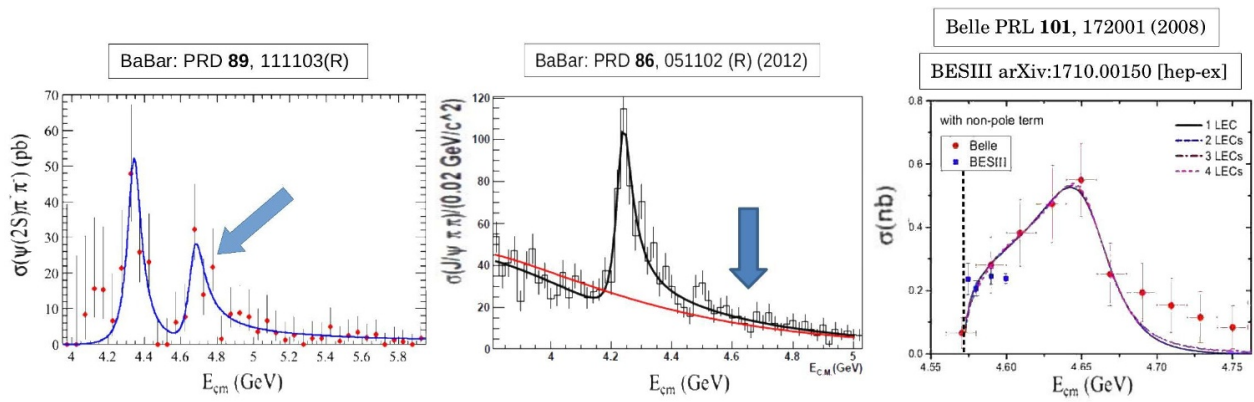

Figure 4. Cross section of the the $Y(4660)$ process of interest: $\pi^{+} \pi^{-} \psi(3686)$ on the left, $\pi^{+} \pi^{-} J / \psi$ in the middle and $\Lambda_{C} \bar{\Lambda}_{C}$ on the right.

tracker with Cylindrical triple-GEM technology will increase the spatial resolution of the secondary vertex needed for the $\Lambda_{C}$ reconstruction. The top-up injection will be implement to keep the beam current nearly constant so that the integrated luminosity can be improved of $20-30 \%$. Finally the beam energy of BEPCII will be increased up to $2.45 \mathrm{GeV}$ in order to study the $\Lambda_{C} \bar{\Lambda}_{C}$ line-shape.

\section{Conclusion}

A large number of $Z$ states has been discovered in charmonium and open-charm decays. Masses and widths are compatible. Isospin triplet has been established for all the decays. Quantum number $\mathbf{J}^{P}=1^{+}$has been measured in a couple of channels. $Y(4220)$ and $Y(4390)$ have been observed in several decays while the $Y(4660)$ shows a more puzzling behavior. An increase in the beam energy will enable a comparison between BESIII and Belle results about the $\Lambda_{C} \bar{\Lambda}_{C}$ cross section line-shape.

\section{References}

[1] M. Ablikim et al., [BESIII Collaboration] Phys. Rev. Lett. 110, 252001 (2013)

[2] R. Aaij et al., [LHCb Collaboration] Phys. Rev. Lett. 115, 072001 (2015)

[3] K.A. Olive et al., [Particle Data Group] Chin. Phys. C 38, 090001 (2014)

[4] G. Pakhlova et al., [Belle Collaboration] Phys. Rev. Lett. 26, no.26 (2008)

[5] S. Godfrey and S. L. Olsen, Annual Review of Nuclear and Particle Science 58:1, 51-73 (2008)

[6] B. Aubert et al., [BaBar Collaboration] Phys. Rev. Lett. 95, 142001 (2005)

[7] M. Ablikim et al., [BESIII Collaboration] Phys. Rev. Lett. 112, 092001 (2014)

[8] M. Ablikim et al., [BESIII Collaboration] Nucl. Instr. A 614, Issue 3 (2010)

[9] R. T. Kleiv et al., Phys. Pev. D 87, 125018 (2013)

[10] Q. Wang et al., Phys. Rev. Lett. 111, 132003 (2013)

[11] M. Ablikim et al., [BESIII Collaboration] Phys. Rev. Lett. 115, 112003 (2015)

[12] M. Ablikim et al., [BESIII Collaboration] Phys. Rev. Lett. 119, 072001 (2017)

[13] M. Ablikim et al., [BESIII Collaboration] Phys. Rev. Lett. 111, 242001 (2013)

[14] M. Ablikim et al., [BESIII Collaboration] Phys. Rev. Lett. 96, 032004 (2017)

[15] M. Ablikim et al., [BESIII Collaboration] Phys. Rev. Lett. 97, 052001 (2018) 
[16] M. Ablikim et al., [BESIII Collaboration] Phys. Rev. Lett. 115, 222002 (2015)

[17] M. Ablikim et al., [BESIII Collaboration] Phys. Rev. Lett. 112, 132001 (2013)

[18] M. Ablikim et al., [BESIII Collaboration] Phys. Rev. Lett. 115, 182002 (2015)

[19] M. Ablikim et al., [BESIII Collaboration] Phys. Rev. Lett. 118, 092001 (2017)

[20] M. Ablikim et al., [BESIII Collaboration] Phys. Rev. Lett. 118, 092002 (2017)

[21] B. Aubert et al., [BaBar Collaboration] Phys. Rev. D 89, 111103(R) (2014)

[22] G. Pakhlova et al., [Belle Collaboration] Phys. Rev. D 91, 112007 (2015)

[23] M. Ablikim et al., [BESIII Collaboration] Phys. Rev. Lett. 96, 032004 (2017)

[24] B. Aubert et al., [BaBar Collaboration] Phys. Rev. D 86, 051102 (R) (2012)

[25] B. Aubert et al., [BaBar Collaboration] arXiv:0808.1543 [hepex] (2008)

[26] G. Cotugno et al., arXiv:0911.2178v5 [hepph] (2017)

[27] G. Pakhlova et al., [Belle Collaboration] Phys. Rev. Lett. 101, 172001 (2008)

[28] M. Ablikim et al., [BESIII Collaboration] arXiv:1710.00150 [hepex] (2017)

[29] G. C. Rossi and G. Veneziano, Nucl. Phys. B 123, 507 (1977)

[30] G. F. Chew, Nucl. Phys. B 79, 365 (1974) 